\title{
The 2012 and 2014 Olympic Doping Scandals: A Critical Analysis of the Effectiveness of Anti-Doping Mechanisms in Sports Law and the Necessity of Global Unity and Rehabilitation in Enforcing Anti-Doping Measures
}

\author{
Anthony G. A. Shaw \\ Diabetes Research Laboratories, School of Clinical Medicine Prince Charles Hospital Northside Clinical Unit, Faculty of Medicine, \\ University of Queensland, Brisbane, Queensland, Australia
}

Email: anthony.shaw@uq.net.au

How to cite this paper: Shaw, A. G. A. (2019). The 2012 and 2014 Olympic Doping Scandals: A Critical Analysis of the Effectiveness of Anti-Doping Mechanisms in Sports Law and the Necessity of Global Unity and Rehabilitation in Enforcing Anti-Doping Measures. Beijing Law Review, 10, 671-681.

https://doi.org/10.4236/blr.2019.104038

Received: June 24, 2019

Accepted: July 27, 2019

Published: July 30, 2019

Copyright $\odot 2019$ by author(s) and Scientific Research Publishing Inc. This work is licensed under the Creative Commons Attribution International License (CC BY 4.0). http://creativecommons.org/licenses/by/4.0/

\begin{abstract}
This paper critically assesses the effectiveness of Olympic anti-doping measures, will consider the way in which the difficulties inherent in the detection of doping may cause infringements on rule of law principles, and discusses approaches to further encourage a mutually beneficial global culture of cooperation and compliance between nations, which must serve as the fundamental basis for the modern development of anti-doping practices. This paper further highlights how the positive steps Russian bodies have taken in response to this scandal and the tangible improvements made to their regulation of doping behaviors are a positive indicator of sports law's capacity to enact change. Moreover, this paper will argue that an approach to anti-doping that seeks to rehabilitate and encourage positive behaviors is ultimately necessary to ensure international sport's role in peacefully facilitating international cooperation, as approaches that vilify and permanently ostracize states that have been found in violation of anti-doping laws are ultimately ineffective at facilitating compliance.
\end{abstract}

\section{Keywords}

Sports Law, Anti-Doping Law, International Law, Olympic Games, Athletics

\section{Introduction}

\subsection{Anti-Doping at the Olympics}

The Olympics is a major event for global competitive sport and is of great signi- 
ficance in terms of promoting athleticism and encouraging fitness in the global community. The Olympics is also vital to commercial and sponsorship interests and plays a key role in international relations and cultural development. The prevention of doping is of vital importance to the Olympics, as it serves to maintain competitive integrity which is essential for the Olympics to maintain its status and global appeal. The term "Doping" describes prohibited practices within sport relating to substances and methods used to enhance performance (Pound \& Clarke, 2011: p. 133), which could lead to an unfair advantage for doping athletes over their opponents. The current rules against doping are based on the 2005 United Nations Economic, Social and Cultural Organization's International Convention against Doping in Sport, which facilitates the World Anti-Doping Agency through articles 11 and 12 (Serby, 2017: p. 4; UNESCO, 2005). As defined in Articles 1 and 2 of the World Anti-Doping Code, doping includes the presence or use or attempted use of a prohibited substance or its metabolites (World Anti-Doping Agency, 2015). Responses to the problem of doping in the Olympics were escalated following the death of a Danish cyclist from a doping related incident at the 1960 Rome Olympics (Pound \& Clarke, 2011: p. 133). Although doping was considered a potential issue prior to this incident, the death of this cyclist revealed the tangible health risks doping caused to participants in the Olympics, which if left to continue unrestricted, would inevitably lead to further injury. Prevention of doping also preserves the values of ethics, fair play, character and education, respect for self and other participants, and the sense of community that is fundamental to the enjoyment of sport (Pound \& Clarke, 2011: p. 141). It is vital to note that for many participating in sports represented at the Olympics, the competition represents the absolute pinnacle of their field, and widespread doping practices in such a publicized event have a widespread cultural influence. This influence can extend to a lower level of competition, and when left unpunished, can spread the message that doping is a necessary component of athletic success. Widespread doping further has commercial impacts, as doping threatens the universal appeal of sport and the wellbeing of the economic stability of the booming sports marketplace (Healey, 1999: p. 3).

\subsection{Research Background}

The Olympics has global influence as an exhibition of athletic ability and is of immense social, economic and political importance. The political prestige that is associated with a strong Olympic presence leads to a temptation for nations to coerce their athletes to utilize doping practices. In the 2012 London Olympics, and the 2014 Sochi Winter Olympics, athletes were found to have been using prohibited substances to gain an unfair advantage in what the International Olympic Committee has called an "unprecedented attack on the integrity of the Olympic Games and sport" (Ingle, 2017). These incidents follow the ongoing struggle the International Olympic Committee has had to identify and punish doping practices assisted by domestic anti-doping organizations that hold a degree of autonomy. 
The doping committed at the 2012 London Olympics and the 2014 Sochi Olympics is notable as it was an endemic subversion of the Olympic anti-doping rules. This corruption extended beyond states coverage of the scandal centered around such as Russia, with former president of the International Association of Athletics Federations (IAAF) Lamine Diack, being found to have contributed to widespread bribery practices (Gibson, 2015). This unprecedented attack on the anti-doping prevention rules signifies a loophole to the global response against doping, and indicated that further intervention would be necessary to prevent localized corruption. Russia has taken steps to reconcile with the World Anti-Doping Agency, and they no longer dispute the endemic corruption in their doping program for the 2012 and 2014 Olympics. This admission optimistically signals a shift towards a path involving reconciliation with Olympic regulators and that conforms to global standards that combat doping practices. Russian authority figures have argued that feelings of distrust led to the doping practices, with high profile member of the Olympic committee, Vitali Smirnov claiming that, "The general feeling in Russia is that we didn't have a chance," (Ruiz, 2016) indicating that Russian Olympic authorities considered other nations to be disproportionately unregulated when compared with their athletes. Nonetheless, Vitali Smirnov indicated a shift away from doping indicating, "We have to find those reasons why young sportsmen are taking doping, why they agree to be doped," (Lawler, 2016) this intention to shift away from doping practices is a hopeful sign that Russian athletes will move away from doping for future Olympic games, and has been corroborated with a nationwide commitment to overhaul their anti-doping system to satisfy the World Anti-Doping Agency (Ruiz, 2016). It is also a hopeful indicator that undetected doping measures exercised by other states will cease. These measures have led to vastly positive results, and Russia has positively enacted steps to react to this scandal and improve conditions (Ingle, 2019). These steps embody an essential and necessity step for the international community to work cooperatively with Russia and establish neutral bodies to combat corruption in anti-doping practices and encourage mutual compliance.

\section{Anti-Doping Measures and the Necessity of International Cooperation}

\subsection{Legal Analysis of the Current Role of Sports Law in Combating Doping in the Olympics}

When assessing the potential for future anti-doping mechanisms to combat scenarios by nations subverting the World Anti-Doping Code such as the 2012 and 2014 doping scandals, it is vital to critically assess the current protections against doping practices. Currently there are a variety of domestic and international bodies which regulate doping with respect to the Olympics, such as The World Anti-Doping Agency, the International Olympic Committee and the Court of Arbitration for Sport (Duval, 2017: p. 177). These are assisted by domestic bodies 
such as the Russian Anti-Doping Agency, who ideally work collaboratively to identify and prevent doping practices.

Doping in the Olympics is regulated by the World Anti-Doping Code (WADC) (Duval, 2017: p. 177). The aim of this code is, "to protect the athlete's fundamental right to participate in fair sport and thus promote health, fairness, and equality for athletes worldwide" (Sage Publications, 2011). Its application is widespread, and many non-Olympic sports have taken steps to comply with the WADC. The WADC is legally enforceable and holds significant weight as it has been adopted under the jurisdiction of the United Nations Educational, Scientific and Cultural Organization, giving the convention tangible influence over governments at an international level (Pound \& Clarke, 2011: p. 135). The WADC is assisted in domestic endeavors by national bodies, eg the Australian National Anti-Doping Scheme, which aim to ensure that sporting administration bodies adopt anti-doping policies complying with WADC (Australian Sports Anti-Doping Authority Act 2006 (Cth) s9; Australian Sports Anti-Doping Authority Regulations 2006 (Cth) sch 1 art 1.01). This dependence on domestic mechanisms, such as the Australian National Anti-Doping Scheme, is not ideal, as in international events with significant domestic stakes such as the Olympics there is a significant conflict of interest. However, domestic regulation by nations is essential as the World Anti-Doping Agency does not have the funds necessary to consistently regulate every participant during training periods. Despite its dependence on domestic groups to monitor athletes, the World Anti-Doping Agency has a global reach as it is funded by the Olympic movement and serves to monitor compliance, scientific research, education, outreach, and out of competition testing (Sage Publications, 2011).

Early on in anti-doping interventions, scientists carried out the testing with little attention paid to the legal framework due to the paternalistic nature of sporting bodies. As commercial interests and the economic clout of individual athletes increased, there was a change to the legalistic approach which involved more onerous practices (Pound \& Clarke, 2011: p. 135). As the 2012 and 2014 doping scandals reveal, even greater attention must be given to domestic agencies carrying out anti-doping schemes to encourage domestic compliance. Despite the massive infringement testing outside of competition periods holds upon the autonomy of athletes, the competition focused testing introduced at the 1968 Olympic Games did not address drugs taken during training periods that could provide residual advantages such as anabolic steroids (Pound \& Clarke, 2011: p. 134). The immediate response to this was the development of longitudinal testing at intervals for these therapies, and this form of testing continues to be necessary to prevent modern doping practices.

Doping violations impute strict liability, and the prosecution is not needed to prove any intent to the doping process (Thorpe, et al. 2013: p. 289). In Foschi $V$ Fina a small white pill was dissolved into edible substances, and the athlete and her managers and coaches denied knowledge of the positive test result (Thorpe, et al. 2013: p. 291). On appeal to the Court of Arbitration for sport the panel 
noted that, "In doping cases it would be practically impossible for a sports federation to prove how a banned substance arrived in the athlete's body ... Any such requirement would be the end of any meaningful fight against doping" (Foschi $V$ Fina (CAS/15S6) 41-42). Any requirement to prove intent would undermine the effectiveness of Olympic anti-doping practices as it would render the policy near-worthless. This precaution is necessary to impede states from doping their athletes without the athletes' knowledge.

Findings of possession or use of prohibited substances have strict liability attached, and it is not necessary to demonstrate intent, fault, negligence or knowing use on the part of the athlete (Pound \& Clarke, 2011: p. 146; World Anti-Doping Agency, 2015, art. 2.1.1, 2.2.1). The attribution of strict liability, whilst having the potential to be highly unfair to athletes, is essential to disincentivize the development of a sports culture which would encourage management staff to take advantage of their athletes and to administer performance enhancing drugs without their knowledge. Previous cases have involved coaches giving athletes prohibited substances mixed with food and drink without their knowledge ( $\mathrm{CV}$ (FINA), CAS 95/141 215), as well as team doctors and coaches prescribing medication without consulting the prohibited substances list (National Wheelchair Basketball v International Paralympic Committee, CAS 95/122). The use of strict liability in regulating anti-doping management is essential because of the immense authority that coaching and management staff, as well as political entities, possess over their Olympic athletes, and provides necessary encouragement to ensure that staff are compliant with the regulations.

As illustrated by the United States Anti-Doping Agency $v$ Collins, circumstantial evidence of doping plays a heightened role in detecting and punishing doping practices, in cases where drugs such as the designer steroid tetrahydrogestrinone could not be identified under testing conditions until 2003 (USADA V Collins AAA No 3019000658 04). Additional circumstantial evidence such as blood tests for testosterone levels, blood tests showing elevated hematocrit and haemoglobin, and evidence such as statements and documents from the organization's files were used to identify the doping practice despite the undetectability of the substance (Thorpe, et al. 2013: p. 298; USADA $v$ Collins $A A A$ No 3019000658 04). Due to the difficulties anti-doping agencies face when combating a consistently changing climate of doping materials (Baxter $\mathrm{V}$ International Olympic Committee (IOC), award of 15 October 2002; Squizzato V FINA, CAS 2005/A/830; Puerta V ITF CAS 2006/A/1025), it is necessary that the role of circumstantial evidence in these cases is more prominent than in standard civil and criminal cases. This was further illustrated in United States Anti-Doping Agency $v$ Lance Armstrong, where evidence from Armstrong's former teammates, as well as banking and accounting evidence, were essential to ascertain his use of and his provision to his teammates of performance enhancing drugs (United States Anti-Doping Agency, 2012), despite a lack of a positive test result. The preceding lack of action taken against him allowed him to participate in doping culture and had, "enforced and re-enforced it" (United States An- 
ti-Doping Agency, 2012). Through this, Lance Armstrong and other cyclists from the United States were revealed to have utilized doping practices to win highly important international competitions such as the Tour DeFrance (Weston, 2017: pp. 97-98). The doping was unable to be directly detected on his samples due to his intermittent hiding from testers, as well as his use of blood transfusion to raise his haematocrit, which was undetectable at that point in time. Ultimately his detection was due to the presence of strict liability in ascertaining the doping behaviors in team based activities, alongside Article 3.2 of WADC which allows admissions and reliable evidence. While strict, these provisions are essential in enforcing anti-doping practices (World Anti-Doping Agency, 2015, art. 3.2), and as such are critical to support the further combating of doping practices at the Olympics.

\subsection{The Necessity for International Cooperation in Combating Doping}

The WADC is universally applicable and is supported by the constituents of the United Nations. As a result, the success of the World Anti-Doping Association (WADA) in regulating the Olympics has been dependent on a unified international community that is willing to participate and self-regulate their athletes (Hunt, 2011: p. 136). However, while each national federation for the Olympics is obliged to adopt an anti-doping policy complying with the WADC, these federations are often reluctant to relinquish their own jurisdiction over their athletes and therefore often maintain their own anti-doping policy and internal panels. Furthermore, the lack of international cooperation in these processes can result in inconsistent adoptions and applications of the WADC, which can involve full refusal to adopt certain provisions (Thorpe, et al. 2013: p. 280). This lack of cooperation is an extreme impediment to anti-doping efforts and played a key role in the doping scandals. Moreover, the need for international cooperation is further highlighted due to the means in which the normative basis of sports law is dependent upon compliance with international agreements between bodies in order to enforce its legal doctrines (Beloff, Kerr and Demetriou, 1999: p. 5).

The necessity for international cooperation is made clear by the means in which the 2012 and 2014 doping scandals signify that increased international fragmentation, and willingness to bypass the WADA regulations, require international cooperation to maintain oversight over national Olympic associations. This international cooperation will ensure that they are behaving in good faith, and are not taking advantage of the WADC's dependence on self-regulation. Moreover, the 2012 and 2014 doping scandals were not the first time a region has attempted to circumvent doping detection practices, for example, the Olympic Committee in East Germany played a significant role in assisting athletes to circumvent drug testing (Thorpe, et al. 2013: p. 275; Yesalis \& Cowart, 1998: p. 76), and these efforts reflect a persistent danger of regional bodies attempting to circumvent the rules. This ongoing risk of violation reveals the necessity of in- 
ternational cooperation to combat doping through consistent international pressure and facilitating a fairly applied international culture that encourages compliance. For example, positive steps have been taken following the doping scandals, and the Russian Anti-Doping Agency has been monitoring and disqualifying athletes who were found to be doping (Kiril, 2017), indicating that mutual co-operation between states has positive results in encouraging compliance. Moreover, further steps to increase accountability and cooperation between nations that are currently being taken, such as the anti-doping agreement the Australian Sports Drug Agency struck with the Chinese Olympic Committee's Anti-Doping Commission to further the development of frequent testing (Thorpe, et al. 2013: p. 275), reflect the significant benefits mutual agreements between countries can form to regulate doping. When considering the WADA's limited resources, the continuation and further development of mutual accountability between nations is essential to preserve the integrity of Olympic anti-doping practices and prevent scenarios such as these doping scandals from occurring in the future. Facilitating further cooperation through anti-doping agreements between Australian, Chinese, Russian, and all state bodies involved in international sport is ultimately necessary to ensure a fair playing field and assist in facilitating a global culture in sport of compliance.

\subsection{Consequences for Those Caught Doping}

Anti-doping rule violations during the Olympics can be quickly acted upon as violation of the provisions mandates automatic disqualification of the result obtained and the forfeiture of any prizes if it is an individual sport (World Anti-Doping Agency, 2015, art. 9). Moreover, all competitive medals, points, and prizes are rendered forfeited from the date of the positive sample collection (World Anti-Doping Agency, 2015, art. 10.8). These penalties are vital in scenarios such as the 2012 and 2014 doping scandals as they reduce the prestige gained from Olympic victories and "shame" states into voluntary compliance with the international protocol. As such, they further impose a period of ineligibility on athletes, with the first violation arising from the use or possession of prohibited substances or methods being 2 years (Pound \& Clarke, 2011: p. 148; World Anti-Doping Agency, 2015, art. 10.2). The penalty imposed on individuals through the ineligibility periods are strong deterrents for nations attempting to subvert the anti-doping rules, as barriers for their athletes toward competitive participation in their field of sport can cause significant damage to their potential to compete and can reduce domestic competition. Furthermore, the sanctions are further strengthened as they become significantly more severe for repeat violations and can range up to lifetime ineligibility from the sport (World Anti-Doping Agency, 2015, art. 10.7.1). The sanctioning of individual athletes is a significant deterrent because ineligibility has far reaching consequences, and can result in the athlete being unable to participate in any competition or activity authorized by any organization that has adopted the WADC during their period of ineligibility (Pound \& Clarke, 2011: p. 151). Furthermore, consequences of 
doping can cause a major detriment to entire teams, as if more than two members of a team are found to have committed an anti-doping rule violation during an event period, the ruling body imposes sanctions on the whole team, such as losing points, disqualification, or other sanctions (World Anti-Doping Agency, 2015, art. 11.2). Although these restrictions appear harsh, especially as entire teams may be disqualified due to the actions of a few, they are essential to ensuring that teams deter their members from the use of prohibited materials. These rulings encourage Olympic teams to foster a culture of compliance and encourage the monitoring of the use of substances by athletes. Furthermore, if utilized fairly, they are capable of providing mechanisms to reform individual athletes who commit slight violations rather than punish entire countries for the actions of individual athletes.

However, for these sanctions on individuals and teams to be granted in a fair environment, it is important for there to be mechanisms of appeal so that procedural fairness is upheld. In common law, where an action will deprive a person of a right or interest, a person should be granted an opportunity to challenge the adverse action and have an opportunity to persuade the decision maker (Blair, 1999: p. 885). Therfore, approaches to doping disputes must embody this notion of procedural fairness. To achieve this goal, the IAAF ensures that the accused has a right to a proper hearing by using a three step process involving: 1) an immediate suspension, followed by 2) an opportunity for hearing, followed by 3 ) ineligibility from competition (Blair, 1999: p. 887; International Association of Athletic Federations, 1999, rule 59). Within this process, concepts such as natural justice, unbiased decision making, and proportionate sanctions are all relevant when deciding how doping is handled, as the laws surrounding doping put forth by WADC are not separate from general legal systems (Pound \& Clarke, 2011: p. 138). Furthermore, when a decision has violated principles of natural justice, the decisions made by a court or professional disciplinary tribunal are irrebuttable evidence of those facts against the athlete (World Anti-Doping Agency, 2015, art. 3.2.3). This helps to ensure that athletes are capable of being treated fairly under sports law, and that important principles of procedural fairness such as the right to a fair hearing and the right to an unbiased decision are utilized in ensuring a just outcome (Bedford \& Weeks, 1999: p. 156). Although this right to appeal potentially causes doping athletes at the Olympics to overturn their penalties, this degree of procedural fairness is vital to ensuring confidence in the anti-doping process and does not significantly hinder international anti-doping efforts. These mechanisms to reform individual athletes who have violated anti-doping laws require international cooperation in ensuring that athletes from each country are fairly treated and given avenues to have their perspective heard. Moreover, approaches that unfairly target athletes from individual nations for political purposes are ineffective as they undermine confidence in anti-doping law's capacity for operating in a manner which is fair and just. The failure of anti-doping legal processes in detecting Lance Armstrong's long term misconduct reveals that the athletes from coun- 
tries such as the United States have previously not been sufficiently investigated.

\subsection{Recent Positive Improvements by Russian Bodies}

In 2019 the Russian International Paralympic committee found that Russia had made significant reform and met 69 of its 70 reinstatement criteria (Ingle, 2019). Russia has further demonstrated voluntary fulfillment of the World Anti-Doping Agency's requirements regarding allowing access to the data and samples that were held at the Moscow Laboratory (Gerdo, 2019). These improvements are highly valued as Russia's continued involvement and support for international sport is an important and necessary facilitator for global cooperation.

\section{Conclusion}

Despite cases such as the 2012 and 2014 Olympic doping scandals having a delayed response, the current harsh legal mechanisms for combating doping in the Olympics are necessary in order to create a level playing field. The doping at the 2012 and 2014 Olympics reflects the ongoing struggle against states that anti-doping agencies have encountered throughout their development and illustrates the importance of national and regional cooperation in the implementation and management of anti-doping practices. The Russian Olympic Committee's response and move towards greater conformity with WADA are a positive indicator of the potential for Russian athletes to participate in future Olympic events in a way that embodies the Olympic aim for fair competition. Moreover, the shift that the Russian Anti-Doping Agency has made to increase cooperation with the WADA is a reminder of the tremendous pressure that the Olympics can exert over national anti-doping agencies with non-conforming tendencies. International partnerships such as the Australian Sports Drug Agency and Chinese Olympic Committee's Anti-Doping Commission's mutual commitment to transparency in combating doping are a positive step forward toward nations holding each other accountable, and more partnerships of this type should be encouraged to foster the mutual respect necessary to create a fair, inclusive, and unifying Olympic games that celebrate competitive integrity. The success of events such as the Olympics is dependent on its role as a facilitator for international collaboration, and the positive efforts made by Russian bodies in response to these scandals strongly reveal that positive encouragement for reform in states that have been found in breach of regulations is a far stronger motivator than vilification and ostracization.

\section{Conflicts of Interest}

The author declares no conflicts of interest regarding the publication of this paper. 


\section{References}

Australian Sports Anti-Doping Authority Act 2006 (Cth).

Australian Sports Anti-Doping Authority Regulations 2006 (Cth).

Baxter v International Olympic Committee (IOC) (2002). Award of 15 October 2002.

Bedford, N., \& Weeks, G. (1999). Doping in Sport: What Role for Administrative Law? In M. Beloff, T. Kerr, \& M. Demetriou (Eds.), Sports Law (pp. 147-168). Oxford: Hart Publishing Ltd.

Blair, F. (1999). Procedural Fairness in Doping Disputes. University of New South Wales Law Journal, 22, 885-892.

CV (FINA), CAS 95/141 215.

Duval, A. (2017). The Russian Doping Scandal at the Court of Arbitration for Sport: Lessons for the World Anti-Doping System. International Sports Law Journal, 16, 177-197. https://doi.org/10.1007/s40318-017-0107-6

Foschi v Fina (CAS/15S6).

Gerdo, V. (2019). Колобков: Россия выполнила все требования WADA по предоставлению доступа к лаборатории. TASS.

Gibson, O. (2015). Russia Accused of "State-Sponsored Doping" as Wada Calls for Athletics Ban. The Guardian.

Healey, D. (1999). The Myth of the Level Playing Field in Sport. In M. Beloff, T. Kerr, \& M. Demetriou (Eds.), Sports Law (pp. 3-18). Oxford: Hart Publishing Ltd.

Hunt, T. (2011). Drug Games-The International Olympic Committee and the Politics of Doping, 1960-2008. Austin, TX: University of Texas Press.

Ingle, S. (2017). Russia Banned from Winter Olympics over State-Sponsored Doping. The Guardian.

Ingle, S. (2019). Russia's Paralympic Doping Ban Lifted after IPC Praises "Significant Reforms". The Guardian.

International Association of Athletics Federations (1999). IAAF Official Handbook: FINA Doping Control Rules (1998-1999).

Kiril, Z. (2017). Five Russian Athletes Suspended for Doping RUSADA Says. The Moscow Times.

Lawler, D. (2016). Russian Officials Admit Institutional Doping Conspiracy for the First Time But Claim Government Was Not Implicated. The Telegraph.

National Wheelchair Basketball v International Paralympic Committee, CAS 95/122.

Pound, R., \& Clarke, K. (2011). Doping in Sport. In J. Nafziger, \& S. Ross (Eds.), Handbook on International Sports Law (pp. 133-161). Northampton: Edward Elgar Publishing.

Puerta v ITF CAS 2006/A/1025.

Ruiz, R. (2016). Russians No Longer Dispute Olympic Doping Operation. The New York Times.

SAGE Publications (2011). Encyclopedia of Sports Medicine.

Serby, T. (2017). Sports Corruption: Sporting Autonomy, Lex Sportiva and the Rule of Law. Entertainment and Sports Law Journal, 15, 2. https://doi.org/10.16997/eslj.204

Squizzato v FINA, CAS 2005/A/830.

Thorpe, D. et al. (2013). Sports Law (2nd ed.). Sydney: Oxford University Press.

UNESCO (2005). International Convention against Doping in Sport. 
United States Anti-Doping Agency (2012). Reasoned Decision of the United States Anti-Doping Agency on Disqualification and Ineligibility.

http://d3epuodzu3wuis.cloudfront.net/ReasonedDecision.pdf

USADA v Collins AAA No 301900065804.

Weston, M. (2017). The Regulation of Doping in U.S. and International Sports. In M. A. McCann (Ed.), The Oxford Handbook of American Sports Law (pp. 83-110). Oxford: Oxford University Press. https://doi.org/10.1093/oxfordhb/9780190465957.013.4

World Anti-Doping Agency (2015). World Anti-Doping Code.

Yesalis, C., \& Cowart, V. (1998). The Steroids Game: An Expert's Inside Look at Anabolic Steroid Use in Sports. Champaign, IL: Human Kinetics. 